Recepción: 06 / 10 / 2016

Aceptación: 27 / 01 / 2017

Publicación: 29 / 04 / 2017

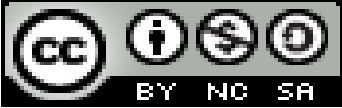

Ciencias de la salud

Artículo de investigación

\title{
Algunas circunspecciones acerca de la salud ocupacional
}

\author{
Some circumspections about occupational health
}

\section{Alguns circunspecciones sobre saúde ocupacional}

\author{
José G. Benalcázar-Game \\ josebenalcazarg@ug.edu.ec \\ Raul G. Castro-García \\ raulcastrog@ug.edu.ec \\ Mary D. Vélez-Almea ${ }^{\text {II }}$ \\ dayi282005@gmail.com
}

Correspondencia: josebenalcazarg@ug.edu.ec

'Magister en Diseño Curricular, Licenciado en Ortesis y Prótesis, Tecnólogo Médico en Ortesis y Prótesis, Docente de la Universidad Estatal de Guayaquil, Guayaquil, Ecuador.

"Magister en Diseño Curricular, Licenciado en Terapia Respiratoria, Tecnólogo Médico en Terapia Respiratoria, Diploma Superior en Diseño Curricular por Competencias, Diploma Superior en Docencia Universitaria, Docente de la Universidad Estatal de Guayaquil, Guayaquil, Ecuador.

"'Magister en Educación Superior, Diploma Superior en Docencia Universitaria, Licenciada en Terapia Ocupacional, Docente de la Universidad Estatal de Guayaquil, Guayaquil, Ecuador. 


\title{
Resumen
}

La salud ocupacional o laboral y la prevención de riesgos, es un elemento imprescindible para que los empleados mejoren no sólo la salud, sino también el bienestar y satisfacción de los trabajadores. A tales efectos se realizó el presente estudio con la finalidad de puntualizar algunos aspectos relacionados con la salud ocupacional: definición, importancias, beneficios y resultados de diferentes investigaciones científicas realizadas en diferentes centros laborales, lo cual permitió llegar a importantes conclusiones.

Palabras clave: salud; salud ocupacional.

\begin{abstract}
Occupational or occupational health and risk prevention, is an essential element for employees to improve not only health, but also the well-being and satisfaction of workers. To this end, the present study was carried out with the aim of highlighting some aspects related to occupational health: definition, importance, benefits and results of different scientific research carried out in different labor centers, which led to important conclusions.
\end{abstract}

Keywords: health; occupational health.

\section{Resumo}

Ocupacional ou saúde ocupacional e prevenção de riscos é um elemento essencial para os funcionários para melhorar não só a saúde, mas também o elemento de satisfação de bem-estar e trabalhador. Para o efeito, o presente estudo, a fim de apontar alguns aspectos da saúde ocupacional foi feito: definição, importâncias, benefícios e resultados de várias pesquisas científicas em diferentes locais de trabalho, o que permitiu chegar a conclusões importantes.

Palavras chave: saúde; saúde Ocupacional.

\section{Introducción}

Desde su aparición sobre la tierra, el hombre debe utilizar parte de su energía en actividades tendientes a modificar la naturaleza circundante, con el fin de satisfacer sus necesidades y la de la sociedad en la que vive. Es decir, el hombre necesita trabajar para alcanzar una vida sana productiva y feliz. ${ }^{1}$

En ese mismo sentido ese trabajo, que al permitirle alcanzar sus objetivos se transforma en un medio indispensable para la realización individual y grupal, no siempre está exento de riesgo para 
la propia existencia. Fácil es visualizarlo si nos imaginamos al hombre primitivo que, en busca de su sustento debía enfrentar un sinnúmero de peligros, que hasta le costaban su vida en no pocas ocasiones, ya sea por las características del medio o de la actividad mediante la cual con rudimentarias herramientas procuraba su alimento. ${ }^{1}$

La urbanización, la industrialización, las nuevas formas de gestión de los medios de producción, las ciencias y la tecnología, trajo aparejado mejoras en la producción de bienes y servicios, que permitió a las poblaciones un acceso más fácil a ellos, aunque todavía hoy con profunda desigualdad, una mejoría en la expectativa y calidad de vida, pero los riesgos presentes en el trabajo para la salud de las personas, si bien con distintas incidencias, continúan en nuestros días según la Organización Internacional del Trabajo. ${ }^{1}$

El trabajo en cualquiera de sus formas, ya sea este remunerado o no, es necesario para la vida humana pero en muchos casos es saludable, ese trabajo, capaz de dañar la propia salud de quienes la realizan de forma individual o colectiva. Aparece de esta forma el trabajo como uno de los determinantes del estado de salud. ${ }^{2}$

La importancia de la salud laboral, ha estado presente desde tiempos inmemorables, al ser el trabajo una actividad sustantiva de los seres humanos. De acuerdo con la definición global adoptada por el Comité Mixto de la Organización Internacional del Trabajo (OIT) y de la Organización Mundial de la Salud (OMS), en su primera reunión en 1950, y revisada en su duodécima reunión en 1995, la finalidad de la salud en el trabajo consiste en lograr la promoción y mantenimiento del más alto grado de bienestar físico, mental y social de los trabajadores en todas las labores; prevenir todo daño causado a la salud de éstos por las condiciones de su trabajo; protegerlos, en su empleo, contra los riesgos resultantes de agentes perjudiciales a su salud; colocar y mantener al trabajador en un empleo adecuado a sus aptitudes fisiológicas y psicológicas y, en suma, adaptar el trabajo al hombre y cada hombre a su actividad (OIT, 2003). ${ }^{2}$

Según la Organización Mundial de la Salud (OMS), la salud ocupacional se define como una actividad multidisciplinaria que controla y realiza medidas de prevención para cuidar la salud de todos los trabajadores, esto incluye enfermedades, cualquier tipo de accidentes y todos los factores que puedan llegar a poner en peligro la vida, la salud o la seguridad de las personas en sus respectivos trabajos. ${ }^{3}$ 
Como principal objetivo, la salud ocupacional genera y promueve que el trabajo sea sano y seguro. Para esto, protege a los trabajadores de la posibilidad de que exista un riesgo en el ambiente laboral para su salud o bienestar. A su vez, está relacionado analizar el medio ambiente y adaptarlo a las condiciones tanto físicas como psíquicas de los trabajadores. Es por esta razón que se establecieron tres objetivos principales de la salud ocupacional. ${ }^{3}$

En primer lugar, se deberá mantener y promover la salud de los empleados, así como también la capacidad que posea cada uno. Las condiciones de trabajo deberán ser lo suficientemente óptimas para favorecer la salud y el bienestar de los mismos. Por último, se hará énfasis en crear sistemas organizacionales para favorecer la salud y la seguridad en el lugar de trabajo, se deberá promover un clima positivo en la organización, se buscará alcanzar una mayor eficiencia y así poder optimizar la productividad de cada empresa. ${ }^{3}$

\section{Funciones de la salud ocupacional}

- Investigar las causas que afectan la salud de los trabajadores.

- Proponer medidas y el desarrollo de actividades que procuren y mantengan la salud en los lugares y ambientes de trabajo.

- Participar en actividades de capacitación en salud ocupacional.

- Colaborar en el análisis de los accidentes de trabajo y enfermedades profesionales e indicar las medidas correctivas a que haya lugar para evitar su ocurrencia.

- Promover la elaboración de planes de trabajo, por parte del comité al interior del programa de salud ocupacional, con el fin de hacer efectivo el tiempo disponible para el cumplimiento de sus funciones. ${ }^{4}$

\section{Salud de los trabajadores}

Los riesgos para la salud en el lugar de trabajo, incluidos el calor, el ruido, el polvo, los productos químicos peligrosos, las máquinas inseguras y el estrés psicosocial provocan enfermedades ocupacionales y pueden agravar otros problemas de salud. Las condiciones de empleo, la ocupación y la posición en la jerarquía del lugar de trabajo también afectan a la salud. Las personas que trabajan bajo presión o en condiciones de empleo precarias son propensas a fumar más, realizar menos actividad física y tener una dieta poco saludable. ${ }^{5}$ 
Además de la atención sanitaria general, todos los trabajadores, y particularmente los de profesiones de alto riesgo, necesitan servicios de salud que evalúen y reduzcan la exposición a riesgos ocupacionales, así como servicios de vigilancia médica para la detección precoz de enfermedades y traumatismos ocupacionales y relacionados con el trabajo. ${ }^{5}$

Las enfermedades respiratorias crónicas, los trastornos del aparato locomotor, las pérdidas de audición provocadas por el ruido y los problemas de la piel son las enfermedades ocupacionales más comunes. ${ }^{5}$

\section{Importancia y beneficios de la salud ocupacional}

La salud laboral en el sentido amplio del término, tal como lo define la Organización Mundial de la Salud (OMS), no es solamente la ausencia de enfermedades, sino que se trata de un estado de completo bienestar físico, mental y social. ${ }^{6}$

Esto significa que al hablar de salud laboral, no nos referimos exclusivamente a las afecciones o a las posibles enfermedades profesionales que puedan padecer los trabajadores, sino que se trata de un concepto mayor, directamente relacionado con el bienestar y la satisfacción en el puesto de trabajo. ${ }^{6}$

Precisamente en la amplitud del concepto radica su gran importancia, puesto que la salud laboral es el principal nexo de unión entre el empleado y la empresa. Hay que tener en cuenta que los profesionales y empleados, es decir, el capital humano es el principal valor de las empresas y pieza imprescindible para lograr unos altos niveles de calidad y productividad. ${ }^{6}$

Ante la situación planteada las ventajas y beneficios de la promoción de la salud laboral y la prevención de riesgos desde las empresas, que la inversión que se pueda realizar en formación o medidas preventivas se recupera muy rápida y fácilmente, las ventajas más destacadas son:

- Se aumenta la productividad y rentabilidad de las empresas o servicios.

- Se disminuye el ausentismo laboral.

- Mejora no sólo de la salud, sino también del bienestar y satisfacción de los trabajadores.

- Se fomentan los vínculos y la fidelidad entre el trabajador y su empresa, evitando la rotación y reteniendo los profesionales con mayor talento. 
- Se mejora el clima laboral, la motivación y la participación.

- Se mejora la imagen corporativa de la empresa o servicios tanto a nivel interno (entre los propios trabajadores) como externo (entre los clientes y la sociedad en general).

- La sociedad en su conjunto se beneficia con una disminución de los costes sociales, sanitarios y de seguridad social, así como en concepto de indemnizaciones por accidente. ${ }^{6}$

En los dos últimos decenios, pese a algunos avances muy importantes realizados por organismos internacionales, gobiernos, empresas, sindicatos y universidades, se considera que el número de accidentes mortales, lesiones y enfermedades ocupacionales sigue siendo inaceptablemente elevado. En los países en desarrollo están aumentando los riesgos para la salud, como consecuencia de la rápida industrialización y la globalización, mientras que los países desarrollados tienen problemas relacionados con el incremento del estrés en el trabajo y el envejecimiento de la población. No cabe duda de que, sin medidas preventivas adecuadas para promover y proteger la salud de los trabajadores, los costos de la salud relacionados con el trabajo y el sufrimiento humano se acrecentarán en el nuevo milenio. ${ }^{7}$

En el presente acápite de nuestro trabajo, nos proponemos presentar los resultados de una revisión documental de investigaciones realizadas en diferentes centros laborales.

Viveros R. ${ }^{8}$ y colaboradores, realizaron un estudio acerca de las Condiciones de Salud y Trabajo en el Comercio Informal del Municipio de Popayán, Colombia. Resultados: de los 883 comerciantes informales incluidos en el estudio, un total de 568 individuos $(64,3 \%)$ fueron mujeres, de las cuáles 80,8\% ganan menos de un SMLV, el 39,2\% son cabeza de familia y solo el 2,99\% tienen nivel de escolaridad superior. La mayor parte pertenecen al régimen subsidiado (47,6\%), pero la protección social cubre solo la salud y no pensiones $(0,3 \%)$ ni riesgos profesionales $(0,8 \%)$. Más del $82,2 \%$ son sedentarios, consumen alcohol en un $54,1 \%$ y se logró detectar tabaquismo en un porcentaje pequeño (10,6\%). Un 51,5\% refirió tener una buena condición de salud, seguido por un $26,8 \%$ en regulares condiciones. En el entorno laboral se encontró que, la población se expone a diferentes riesgos como: ergonómicos, físicos, químicos, de seguridad, psicosocial e inseguridad ciudadana. Conclusiones: Las condiciones de salud de la población evaluada son buenas a pesar de que no todos se encuentran afiliados a un sistema general de seguridad integral; sin embargo, las condiciones de trabajo si son precarias. 
Otro estudio realizado por Andrade Jaramillo $\mathrm{V}^{2}$, con respecto a la salud laboral. Investigaciones realizadas en Colombia, la cual tuvo como objetivo dar cuenta de manera general sobre las temáticas relacionadas con la salud laboral, que se han investigado en los últimos años en Colombia, y evidenciar la relevancia que tiene, dentro del estudio de este tema, la investigación e intervención desde las ciencias sociales y de la salud. Se encontró que existe un desarrollo lento, respecto a otros países, en investigaciones sobre el tema, pero que refleja la influencia de las nuevas tendencias del trabajo en la temática. Se concluye que debe promoverse la realización de una investigación que contemple metodologías mixtas y de corte longitudinal para una mayor comprensión de los fenómenos, así como fortalecer la conexión entre la academia y las organizaciones a fin de realizar investigaciones que den soluciones a problemáticas específicas de la relación salud-trabajo.

Según datos obtenidos en la investigación realizada, por Sánchez Jacas I ${ }^{9}$, en el Departamento de Control de Vectores del Policlínico Docente "Carlos Juan Finlay" de Santiago de Cuba, en el período enero-mayo del 2013, con el propósito de evaluar la calidad del trabajo de los operarios A, B y los jefes de brigada, a partir de sus conocimientos para un eficiente desempeño en la lucha contra el Aedes aegypti, y sobre la base de criterios, indicadores y estándares elaborados a los efectos. Se obtuvo que los trabajadores no poseían los conocimientos necesarios ni estaban satisfechos con las condiciones y desempeño laborales, pues los criterios establecidos resultaron inadecuados en la totalidad de ellos; en cuanto a la satisfacción de los moradores, solo 8 criterios fueron adecuados. De esta manera, pudo concluirse que los operarios y jefes de brigada no dominaban las actividades diarias de la campaña antivectorial.

En un estudio efectuado en la Dirección Municipal de Salud de Arroyo Naranjo, La Habana, Cuba, con el objetivo de caracterizar la morbilidad con incapacidad laboral en una población trabajadora, se realizó un estudio de tipo transversal en un total de 56 trabajadores. Se revisaron los certificados médicos emitidos a trabajadores con el diagnóstico de hipertensión arterial en un área de salud. El mayor número de días de incapacidad temporal para el trabajo se manifestó en el grupo de edad de 40 a 49 años, seguido por el de 50 a 59 años. Predominaron las categorías de servicio y técnicos. Los días otorgados por esta causa tuvieron predominio en los certificados emitidos de hasta 15 días y de 30 días y más. Se concluye que esta enfermedad puede estar relacionada con factores que, de manera independiente o concurrente, incrementan la posibilidad 
de crisis u otros daños con limitaciones temporales o permanentes para trabajar, por lo que se impone establecer acciones de prevención y promoción de salud. ${ }^{10}$

Otros autores plantean, que muchos de los trabajadores afectados experimentan pérdidas considerables, al estar expuesto a ruidos superiores a los $25 \mathrm{~dB}$. La dificultad auditiva causada por el ruido puede tener efectos significativos tanto en su empleo como en sus interacciones sociales y familiares. Los resultados de la investigación realizada en los departamentos del Servicio Estomatológico del Policlínico "Julián Grimau García" presentan niveles superiores de ruido que el normado, lo cual provoca efectos negativos sobre la salud al ocasionar problemas auditivos como la hipoacusia.

\section{Conclusiones}

Es necesario promover las investigaciones en salud ocupacional, ya que éstas pueden repercutir con sus resultados en políticas organizacionales que promuevan el bienestar de las personas en el trabajo, disminuyan costos, aumenten la productividad, fomenten la competitividad organizacional, incrementen la satisfacción en el trabajo.

Por otra parte los programas de gestión de la salud en el lugar de trabajo deberán ser asequibles y presupuestarse de forma acorde con la capacidad del lugar de trabajo y las necesidades prioritarias.

\section{Referencias bibliográficas}

1- Nieto H A. Salud laboral. Capítulo del libro medicina y salud pública 1999. Pág. Introducción a las condiciones y medio ambiente del trabajo. OIT. Ginebra 1987.

2- Jaramillo A, Gómez V, Carolina I. Salud laboral. Investigaciones realizadas en Colombia. Pensamiento psicológico 2008 [citado 15 septiembre 2016]; 4. (10). pp. 9-25. Disponible en: http://www.redalyc.org/articulo.oa?id=80111670002

3- Concepto de salud ocupacional. 2014. [citado 15 septiembre 2016]. Disponible en: http://concepto.de/salud-ocupacional/

4- Funciones salud ocupacional. 2016 [citado 15 septiembre 2016]. Disponible en: http://www.unalmed.edu.co/ copaso/funciones.html 
5- Salud de los trabajadores. OMS. 2017. [citado 15 septiembre 2016]. Disponible en: http://www.who.int/topics/occupational_health/es/

6- Importancia y beneficios de la salud laboral. 2015. [citado 15 septiembre 2016]. Disponible en: http://www.aguaeden.es/acerca-de-nosotros/blog-agua-eden/importancia-y-beneficios-de-lasalud-laboral/

7- Chu C. De una promoción de la salud en el lugar de trabajo hacia la gestión integradora de la salud en el lugar de trabajo: Tendencias y evolución. 2003. [citado 15 septiembre 2016]. Disponible en: www.who.int/occupational_health/publications/newsletter/en/gohnet6s.pdf

8- Viveros J R, Orozco J A, Cruz A M. Condiciones de salud y trabajo en el Comercio Informal del Municipio de Popayán, Colombia. Revista Colombiana de Salud Ocupacional, 2(3), Sept 2012, pp 5-12.

9- Sánchez Jacas I, Delás Jaen E, Suárez Vélez H D. Conocimiento y satisfacción de los trabajadores de la campaña antivectorial contra el Aedes aegypti. Revista medisan 2016 [citado 15 septiembre 2016]; 20 (7). Disponible http://www.medisan.sld.cu/index.php/san/article/view/299/html

10- Jova Linares Y. Hipertensión arterial: una causa de incapacidad temporal. 2010. Revista Cubana de Salud y Trabajo [citado 15 septiembre 2016]; 11(3):41-4. Disponible en: http://bvs.sld.cu/revistas/rst/vol11_3_10/rst06310.htm

11- Espinoza Ormeño Y, Hernández Cazcarra K, Ortega López G, Pilquil Fernández M. Niveles de ruido ocupacional y desempeño audiológico en estudiantes y profesionales de odontología. Universidad de Chile, 2013. Disponible en: http://repositorio.uchile.cl/handle/2250/11681 